\title{
ESTIMATION OF CONTRIBUTIONS TO POPULATION GROWTH: A REVERSE-TIME CAPTURE-RECAPTURE APPROACH
}

\author{
James D. Nichols, ${ }^{1,3}$ James E. Hines, ${ }^{1}$ Jean-Dominique Lebreton, ${ }^{2}$ And Roger Pradel ${ }^{2}$ \\ ${ }^{1}$ US Geological Survey, Biological Resources Division, Patuxent Wildlife Research Center, Laurel, Maryland 20708 USA \\ ${ }^{2}$ Centre d'Ecologie Fonctionnelle et Evolutive/Centre National de la Recherche Scientifique (CEFE/CNRS), \\ 1919 Route de Mende, BP 5051, F-34033 Montpellier, Cedex 01, France
}

\begin{abstract}
We consider methods for estimating the relative contributions of different demographic components, and their associated vital rates, to population growth. We identify components of the population at time $i$ (including a component for animals not in the population at $i$ ). For each such component we ask the following question: "What is the probability that an individual randomly selected from the population at time $i+1$ was a member of this component at $i$ ?" The estimation methods for these probabilities $\left(\gamma_{i}\right)$ are based on capture-recapture studies of marked animal populations and use reverse-time modeling. We consider several different sampling situations and present example analyses for meadow voles, Microtus pennsylvanicus. The relationship between these $\gamma_{i}$ parameters and elasticities (and other parameters based on projection matrix asymptotics) is noted and discussed. We conclude by suggesting that model-based asymptotics be viewed as demographic theory and that direct estimation approaches be used to test this theory with data from sampled populations with marked animals.
\end{abstract}

Key words: animal population ecology; asymptotic theory of population growth; contributions of demographic components; elasticity; meadow voles; Microtus pennsylvanicus; population growth rate; population projection matrix; reverse-time capture-recapture models.

\section{INTRODUCTION}

Population growth is a process of fundamental interest in the study of animal population dynamics. At any time, $i+1$, in the history of an animal population, it is possible to ask the following question: "What is the composition of this population, $N_{i+1}$, with respect to classes of animals the previous time period, $i$ ?" For example, if we deal with a single patch or subpopulation within a metapopulation system, we might ask what proportion of the subpopulation at time $i+1$ comprises survivors from the same subpopulation at time $i$, and what proportion is instead new animals that were outside the subpopulation at $i$, but immigrated between $i$ and $i+1$. Certainly this kind of question is relevant to the identification of source and sink areas (Pulliam 1988) and to general questions about metapopulation dynamics. Alternatively, we might classify animals by age and ask about the relative contributions of young and adults at time $i$ to the adult population at time $i+1$.

The finite rate of increase of a population between two periods, $i$ and $i+1$, is usually defined as $\lambda_{i}=$ $N_{i+1} / N_{i}$, where $N_{i}$ is the population size for time $i$. Thus, contributions of different classes of animals at time $i$ to $N_{i+1}$ also represent contributions of these classes to population growth, $\lambda_{i}$. In this paper, we show how reverse-time capture-recapture methods (Pollock et al.

Manuscript received 7 June 1999; revised 2 November 1999; accepted 1 December 1999; final version received 3 January 2000.

${ }^{3}$ E-mail: Jim_Nichols@usgs.gov
1974, Nichols et al. 1986, Pradel 1996) can be used to directly estimate such contributions to population size and growth rate. We illustrate the approach with examples, and finally discuss the relationship between these estimated contributions to population growth and other quantities developed to address similar sorts of questions (e.g., sensitivity, elasticity, or relative sensitivity; see Caswell [1989]).

Specifically, we examine three scenarios that differ with respect to the number of subpopulations and the number of age classes considered. For each scenario, we express population change in terms of the relevant demographic components and show the corresponding decomposition of population growth rate. We write the numbers of animals in each component as a binomial or multinomial random variable conditional on $N_{i+1}$ and focus attention on the cell probability parameters. We then turn to estimation of these parameters and consider the use of reverse-time capture-recapture methods for this purpose. Finally, we present an example of the use of these estimators with data from field studies of meadow voles, Microtus pennsylvanicus, at Patuxent Wildlife Research Center, Laurel, Maryland.

\section{One Age, One Stratum \\ Demographic components}

Assume interest in a single, open (birth, death, emigration, and immigration can occur between sample periods) animal population with no age-specificity. We would like to estimate the relative contributions to population growth or change between $i$ and $i+1\left(\lambda_{i}\right)$ of 
two demographic components: (1) surviving animals from the population at time $i$ (denote these as $S_{i}$ ), and (2) new recruits (denote these as $B_{i}$ ). (Note that we follow the convention of referring to $\lambda_{i}$ as population growth rate despite the fact that it can reflect decreases in population size $\left[\lambda_{i}<1\right]$ and stasis $\left[\lambda_{i}=1\right]$, as well as increases $\left.\left[\lambda_{i}>1\right]\right)$. The recruits are animals not in the population at time $i$, but instead result from reproduction and/or immigration, enter the population between times $i$ and $i+1$, and are present at $i+1$. We view population size, $N_{i+1}$, number of survivors, $S_{i}$, and number of new recruits, $B_{i}$, as random variables. Population size at time $i+1$ can be written as the sum of these two demographic components:

$$
N_{i+1}=S_{i}+B_{i} .
$$

We can view these two components $\left(S_{i}\right.$ and $\left.B_{i}\right)$ of $N_{i+1}$ as following a binomial distribution conditional on $N_{i+1}$ and governed by a parameter, $\gamma_{i+1}$, denoting the probability that a member of $N_{i+1}$ is a survivor from the previous period (i.e., a member of $S_{i}$ ). Thus, we can write the probability distribution of $S_{i}$, conditional on $N_{i+1}$, as follows:

$\operatorname{Pr}\left(S_{i} \mid N_{i+1}\right)=\frac{\left(N_{i+1}\right) !}{\left(S_{i}\right) !\left(N_{i+1}-S_{i}\right) !} \gamma_{i+1}^{S_{i}}\left(1-\gamma_{i+1}\right)^{N_{i+1}-S_{i}}$

where $B_{i}=N_{i+1}-S_{i}$.

Based on Eqs. 1 and 2, we can decompose the expectation for population growth rate as follows:

$$
\begin{aligned}
E\left(\lambda_{i}\right) & \approx \frac{E\left(S_{i}\right)+E\left(B_{i}\right)}{E\left(N_{i}\right)} \\
& =\frac{\gamma_{i+1} N_{i+1}+\left(1-\gamma_{i+1}\right) N_{i+1}}{E\left(N_{i}\right)} .
\end{aligned}
$$

We believe that the $\gamma_{i+1}$ parameters provide useful information about the components of population growth. For example, if $\gamma_{i+1}=0.5$, then we can regard survivors from $N_{i}$ and new recruits as equally important to population growth over the interval $i$ to $i+1$. If $\gamma_{i+1}=0.75$, then a member of $N_{i+1}$ is three times more likely to be a survivor from time $i$ than to be a new recruit, and survival within the population can be viewed as three times more important to population growth over the interval $i$ to $i+1$.

These parameters, $\gamma_{i+1}$, can be used to draw inferences about the relative effect of hypothetical changes in the two demographic components on the population growth between $i$ and $i+1$. For example, assume that recruitment had been reduced by proportion $\alpha$ between $i$ and $i+1$, such that recruitment during this interval was really $(1-\alpha) B_{i}$. The proportional change in $\lambda_{i}$ resulting from proportional change $\alpha$ in recruitment is given by $\alpha\left(1-\gamma_{i+1}\right)$. Thus, we can compute the new population growth rate resulting from a proportional reduction in recruitment of magnitude $\alpha$ as $\lambda_{i}[1-\alpha(1$ $\left.\left.-\gamma_{i+1}\right)\right]$.

\section{Estimation}

Capture-recapture studies provide information that can be used to estimate the $\gamma_{i+1}$ parameters. In sampling period $i+1$, we may catch an animal that was captured and marked in some previous period, period $\leq i$. We know that such an animal was a member of $S_{i}$. However, if we catch an unmarked animal at time $i+1$, we cannot be certain of its status at time $i$. The animal could have been in the population before sampling period $i+1$ and not caught, or it could have been a new recruit at $i+1$, consequently not available for capture before $i$ +1 . Estimation of $\gamma_{i+1}$ thus requires consideration of capture probabilities, $p_{i}$ (probability that an animal in the population at $i$ is caught or observed in sampling efforts at $i$ ), and the use of capture-recapture modeling (e.g., Lebreton et al. 1992).

Survival rate estimation under capture-recapture models for open populations, such as the CormackJolly-Seber (CJS) model (Cormack 1964, Jolly 1965, Seber 1965), proceeds by conditioning on releases in earlier time periods and following fates of these animals in later time periods. Pollock et al. (1974) noted that if the capture history data are considered in reverse time order, conditioning on animals caught in later time periods and observing their captures in earlier occasions, then inference can be made about the recruitment process. Specifically, "a backward process with recruitment and no mortality is statistically equivalent to a forward process with mortality and no recruitment", (Pollock et al. 1974:85-86).

Data from capture-recapture studies are typically summarized as capture histories. For example, the capture history 0101100 indicates an animal captured only in periods 2, 4, and 5 of a seven-period study (each element of the row vector represents observation (1) or nonobservation (0) of the animal at a sampling period, where such periods are numbered consecutively beginning at period 1). Standard capture-recapture survival analysis (e.g., Lebreton et al. 1992) proceeds by first conditioning on the period of initial capture of an animal (period 2 for capture history 0101100) and then modeling the subsequent capture history using two kinds of parameters, $\phi_{i}$ (the probability that an animal present in the population at sampling period $i$ survives and is still in the population at period $i+1)$ and $p_{i}$. So conditional on capture in period 2 , the probability associated with the remainder of the above capture history would be: $\phi_{2}\left(1-p_{3}\right) \phi_{3} p_{4} \phi_{4} p_{5} \chi_{5}$, where the $\chi_{i}$ parameters denote the probability of not being seen at any time after $i$, given that the animal is alive at $i$. The $\chi_{i}$ are not really new parameters, in the sense that they are written recursively as a function of subsequent $\phi_{i}$ and $p_{i}$ [in the example, $\chi_{5}$ for a seven-period study can be written as $\left.\left(1-\phi_{5}\right)+\phi_{5}\left(1-p_{6}\right)\left(1-\phi_{6} p_{7}\right)\right]$. Capture history data such as these lead to conditional productmultinomial models from which maximum likelihood 
estimates of the $\phi_{i}$ can be directly obtained (Seber 1982, Lebreton et al. 1992).

Reverse-time capture-recapture analyses (Pollock et al. 1974, Nichols et al. 1986, Pradel 1996) proceed in a straightforward manner by simply reversing the order of the capture history data. If we reverse the time order of the above capture history, we obtain 0011010, where the first zero represents period 7, the second represents period 6 , and the final zero represents period 1 . The reverse-time approach to capture-recapture analysis is to model the reverse capture history data with $p_{i}$ and $\gamma_{i}$, where $\gamma_{i}$ reflects the probability that an animal present in sampling period $i$ was also present in period $i$ -1 (this is the same parameter that was defined previously and used to decompose the population growth rate). The $\gamma_{i}$ thus concern the "survival" of an animal into the previous sampling period. Conditional on final recapture in period 5, reverse capture history 0011010 would be modeled as: $\gamma_{5} p_{4} \gamma_{4}\left(1-p_{3}\right) \gamma_{3} p_{2}\left(1-\gamma_{2} p_{1}\right)$. Such capture history data lead to the same kind of product-multinomial models as standard-time data, and maximum likelihood estimation of the $\gamma_{i}$ proceeds in exactly the same manner as for estimation of $\phi_{i}$ that we have presented.

As noted by Pradel (1996), the software packages (e.g., SURGE [Lebreton et al. 1992], MARK [White and Burnham 1999]) used to estimate $\phi_{i}$ from standardtime capture-recapture data provide estimates of $\gamma_{i}$ when applied to reverse-time data. A similar, but alternative, approach to estimation of $\gamma_{i}$ does not condition on final captures, but is instead based on the full likelihood also introduced by Pradel (1996). Although we have based our recommendations and examples on the conditional approach because of its ease of implementation, it may be that the full likelihood approach of Pradel (1996) (also implemented in MARK) provides slightly more precise estimates in some situations. This topic merits exploration.

In capture-recapture studies animals sometimes die in traps or are not released following sampling for other reasons. We note that when this occurs, it is usually appropriate to define $\lambda_{i}$ as $\lambda_{i}=N_{i+1}^{-} / N_{i}^{+}$, where $N_{i}^{-}$and $N_{i}^{+}$denote population size just before and after sampling at time $i$, respectively. This definition restricts attention to changes in abundance that are not associated with the sampling process. The $\gamma_{i}$ estimated from capture-recapture studies as described here reflect proportional contributions to $N_{i+1}^{-}$and, hence, to $\lambda_{i}$ as defined here. In the example analysis, we assume no losses on capture, but this modified definition of $\lambda_{\iota}$ will be applicable in studies that do have losses.

\section{Example analysis}

We use capture-recapture data from a study of meadow voles, Microtus pennsylvanicus, conducted in oldfield habitat at Patuxent Wildlife Research Center in Laurel, Maryland (also see Nichols et al. 1994). Here we focus on one of eight experimental trapping grids, grid 4A, and use data from the first 11 sampling periods extending from November 1991-May 1993. The grid was a $7 \times 15$ rectangle of trapping stations with adjacent stations within each row or column separated by $7.6 \mathrm{~m}(25 \mathrm{ft})$. The robust design (Pollock 1982) was used, with primary trapping periods occurring at $\sim 8$ wk intervals. For primary periods $1-9$, secondary sampling included five consecutive days of trapping, whereas for periods $10-11$, only two consecutive days were trapped.

A single Sherman live trap containing cotton and baited with rolled oats was placed at each station. The trapping schedule consisted of setting traps one evening, checking them for animals and closing them the following morning, setting them again in the late afternoon, checking them the following morning, etc. Newly captured animals were marked with individually coded monel fingerling tags placed in their ears. If tags of previously marked animals showed signs of pulling out, we applied a new tag on the opposite ear and/or clipped toes. Animals were sexed and masses were measured on each occasion, and external reproductive characteristics were recorded.

For this analysis, we focused on adult and subadult voles (defined as $>22 \mathrm{~g}$ body mass) of both sexes. Both the data set and the analysis are available as Supplementary Materials. We used an open model approach, focusing only on whether or not an animal had been captured at least once at each of the 11 primary trapping periods. The analysis used 874 captures of 451 adult and subadult (hereafter simply termed "adult") animals. As this is a single-age, single-stratum problem, we assessed the fit of the standard Cormack-Jolly-Seber model (Cormack 1964, Jolly 1965, Seber 1965) and found it adequate $\left(\chi_{14}^{2}=19.1, P=0.16\right.$; Pollock et al. 1985, 1990).

We estimated the time-specific rates of population increase (corresponding to approximate two-month periods) using the approach of Pradel (1996). Estimates, $\hat{\lambda}_{i}$, ranged $0.59-1.73$, as the population exhibited apparent increases and declines over the 1.5-yr study period (Table 1). We used reverse-time capture-recapture to estimate the relative contributions of adult survival $\left(\hat{\gamma}_{i}\right)$ and recruitment $\left(1-\hat{\gamma}_{i}\right)$ to population growth between $i-1$ and $i$ (i.e., $\lambda_{i-1}$ and $\gamma_{i}$ both correspond to the interval, $i-1$ to $i$ ). The point estimates of $\gamma_{i}$ ranged $0.40-0.83$, indicating substantial variation in the relative contribution of adult survival to population growth (Table 1). Nevertheless, for all but two intervals, $\hat{\gamma}_{i}>0.5$, indicating a tendency for adult survival to be more important to population growth than recruitment. Recall that recruitment, in this situation, refers to adults that are "new" in period $i$, in the sense that they were not adults on the area in period $i-1$. Such recruits could have resulted from reproduction on the grid or from animals moving onto the grid from outside areas. We distinguish between these two recruitment components in subsequent examples. 
TABLE 1. Estimates of population growth rate $\hat{\lambda}_{i}$ and relative contributions to population growth of adult survival $\left(\hat{\gamma}_{i}\right)$ and recruitment $\left(1-\hat{\gamma}_{i}\right)$ for adult meadow voles trapped on grid 4A, Patuxent Wildlife Research Center, Laurel, Maryland, November 1991-May 1993.

\begin{tabular}{|c|c|c|c|c|}
\hline $\begin{array}{l}\text { Trapping } \\
\text { period }\end{array}$ & Date $\dagger$ & $\hat{\lambda}_{i}\left(\operatorname{SE}\left[\hat{\lambda}_{i}\right]\right) \ddagger$ & $\hat{\gamma}_{i}\left(\operatorname{SE}\left[\hat{\gamma}_{i}\right]\right) \S$ & $1-\hat{\gamma}_{i}\left(\operatorname{SE}\left[1-\hat{\gamma}_{i}\right]\right) \S$ \\
\hline 2 & 1 January 1992 & $1.06(0.362)$ & & \\
\hline 3 & 26 February 1992 & $0.59(0.209)$ & $0.60(0.109)$ & $0.40(0.109)$ \\
\hline 4 & 22 April 1992 & $1.73(0.560)$ & $0.64(0.117)$ & $0.36(0.117)$ \\
\hline 5 & 17 June 1992 & $0.97(0.232)$ & $0.40(0.055)$ & $0.60(0.055)$ \\
\hline 6 & 12 August 1992 & $0.87(0.126)$ & $0.50(0.064)$ & $0.50(0.064)$ \\
\hline 7 & 7 October 1992 & $1.39(0.140)$ & $0.65(0.049)$ & $0.35(0.049)$ \\
\hline 8 & 1 December 1992 & $0.70(0.107)$ & $0.53(0.043)$ & $0.47(0.043)$ \\
\hline 9 & 27 January 1993 & $0.59(0.183)$ & $0.83(0.049)$ & $0.17(0.049)$ \\
\hline 10 & 25 March 1993 & $\ldots$ & $0.70(0.081)$ & $0.30(0.081)$ \\
\hline 11 & 20 May 1993 & $\ldots$ & $0.58(0.109)$ & $0.42(0.109)$ \\
\hline
\end{tabular}

$\dagger$ Midpoint of five-day (periods 1-8) trapping period and first of two-day (9-11) periods.

\$ Estimated directly using the approach of Pradel (1996).

$\S$ Estimated using reverse-time capture-recapture.

We can use these estimates, $\hat{\gamma}_{i}$, to draw inferences about the degree to which population growth would have differed had either recruitment or adult survival been different. For example, $\hat{\gamma}_{10}=0.70$, indicating that an estimated $70 \%$ of the adult population at period 10 were surviving adults from period 9 . The rate of population increase between periods 9 and 10 was estimated to be $\hat{\lambda}_{9}=0.59$, reflecting a decline in adult population size over this interval. What would have been the population growth rate over this interval if adult survival had been $5 \%$ higher or $5 \%$ lower than it actually was (we refer to $\phi_{i} \pm 0.05 \phi_{i}$ and not $\phi_{i} \pm$ $0.05)$ ? We compute the change in growth rate corresponding to a $5 \%$ change in adult survival as 0.05 $\hat{\gamma}_{10}$ or $3.5 \%$ (estimated SE $=0.405 \%$ ). So a $5 \%$ increase in adult survival over the interval between sampling periods 9 and 10 would have increased $\hat{\lambda}_{9}=0.59$ by $3.5 \%$ to a projected $1.035 \hat{\lambda}_{9}=0.61$. Similarly, a $5 \%$ decrease in adult survival would have reduced $\lambda$ to $0.965 \hat{\lambda}_{9}=0.57$.

\section{One Age, Two Strata}

\section{Interest focused on one stratum}

Demographic components.-Assume a sampling situation where we mark and recapture animals at two different study areas (denoted areas 1 and 2) and that animals can move between these two areas. Further assume that our primary interest is in population growth on one of these areas (say area 1). Define $S_{i}^{r s}$ as the number of animals located on area $r$ at time $i$ that are alive on area $s$ at time $i+1$. Using superscripts to denote the study area, we can write the population size on area 1 as the sum of three components:

$$
N_{i+1}^{1}=S_{i}^{11}+S_{i}^{21}+B_{i}^{1} .
$$

The first term on the right-hand side of Eq. 4 represents the number of animals that survived from $i$ to $i+1$ and remained on area 1 . The second term denotes animals present on area 2 at time $i$ that survived until $i$ +1 and moved to area 1 . The final term simply rep- resents the recruitment to area 1 between $i$ and $i+1$, including results of any reproduction on areas 1 and 2, and immigration from locations outside the boundaries of the two study areas. Thus, $B_{i}^{1}=N_{i+1}^{1}-S_{i}^{11}-S_{i}^{21}$. We can model these random variables reflecting the demographic components contributing to $N_{i+1}^{1}$ as a conditional trinomial:

$$
\begin{aligned}
P\left(S_{i}^{11}, S_{i}^{21}, B_{i}^{1} \mid N_{i+1}^{1}\right)= & \frac{\left(N_{i+1}^{1}\right) !}{\left(S_{i}^{11}\right) !\left(S_{i}^{21}\right) !\left(B_{i}^{1}\right) !}\left(\gamma_{i+1}^{11}\right)^{\left(S_{i}^{11}\right)} \\
& \times\left(\gamma_{i+1}^{12}\right)^{\left(S_{i}^{21}\right)}\left(1-\gamma_{i+1}^{11}-\gamma_{i+1}^{12}\right)^{\left(B_{i}^{1}\right)} .
\end{aligned}
$$

The $\gamma_{i+1}^{r s}$ are defined as the probability that an animal present in area $r$ during period $i+1$ was in area $s$ in period $i$. In this simple case of two study areas, $\gamma_{i+1}^{11}$ gives the probability that a member of $N_{i+1}^{1}$ was a member of $N_{i}^{1}$ the previous period, $\gamma_{i+1}^{12}$ is the probability that a member of $N_{i+1}^{1}$ was a member of $N_{i}^{2}$ the previous period, and $1-\gamma_{i+1}^{11}-\gamma_{i+1}^{12}$ represents the probability that a member of $N_{i+1}^{1}$ was in neither area 1 nor 2 at time $i$, but was recruited (either via reproduction or immigration) between times $i$ and $i+1$. The $\gamma_{i+1}^{r s}$ parameters quantify the relative contributions of these different demographic components to the population at time $i+1$ and thus to population growth between $i$ and $i+1$. Based on Eqs. 4 and 5, we can decompose the expectation for population growth rate of area 1 as follows:

$$
\begin{aligned}
E\left(\lambda_{i}^{1}\right) & \approx \frac{E\left(S_{i}^{11}\right)+E\left(S_{i}^{21}\right)+E\left(B_{i}^{1}\right)}{E\left(N_{i}^{1}\right)} \\
& =\frac{\gamma_{i+1}^{11} N_{i+1}^{1}+\gamma_{i+1}^{12} N_{i+1}^{1}+\left(1-\gamma_{i+1}^{11}-\gamma_{i+1}^{12}\right) N_{i+1}^{1}}{E\left(N_{i}^{1}\right)} .
\end{aligned}
$$

The $\gamma_{i+1}^{r s}$ parameters can be used to address questions such as the following: "How would the rate of increase in area $1\left(\lambda_{i}^{1}\right)$ have differed if the contribution from area 2 had been reduced by $25 \%$." Let $\alpha$ be the pro- 
TABLE 2. Estimates of population change $\left(\hat{\lambda}_{i}^{1}\right)$ for patch 1 of a two-patch system and relative contributions to population size and growth of adult survival on patch $1\left(\hat{\gamma}_{i}^{11}\right)$, adult survival and movement from patch 2 to patch $1\left(\hat{\gamma}_{i}^{12}\right)$, and recruitment to patch 1 from outside the two-patch system $\left(1-\hat{\gamma}_{i}^{11}-\hat{\gamma}_{i}^{12}\right)$ for meadow voles trapped on grid 4A, Patuxent Wildlife Research Center, Laurel, Maryland, November 1991-May 1993.

\begin{tabular}{cccccc}
\hline \hline $\begin{array}{c}\text { Trapping } \\
\text { period }\end{array}$ & Date $\dagger$ & $\hat{\lambda}_{i}^{1}\left(\operatorname{sE} \hat{\lambda}_{i}^{1}\right) \ddagger$ & $\hat{\gamma}_{i}^{11}\left(\operatorname{sE}\left[\hat{\gamma}_{i}^{11}\right]\right) \S$ & $\hat{\gamma}_{i}^{12}\left(\operatorname{SE~}\left[\hat{\gamma}_{i}^{12}\right]\right) \S$ & $\begin{array}{c}1-\hat{\gamma}_{i}^{11}-\hat{\gamma}_{i}^{12} \\
\left(\operatorname{SE~}\left[1-\hat{\gamma}_{i}^{11}-\hat{\gamma}_{i}^{12}\right]\right) \S\end{array}$ \\
\hline 2 & 1 January 1992 & $1.25(0.735)$ & $\ldots$ & $\ldots$ & $\ldots$ \\
3 & 26 February 1992 & $0.51(0.360)$ & $0.49(0.126)$ & $0.00(0.000)$ & $0.51(0.126)$ \\
4 & 22 April 1992 & $1.03(0.767)$ & $0.62(0.166)$ & $0.04(0.035)$ & $0.34(0.169)$ \\
5 & 17 June 1992 & $1.36(0.832)$ & $0.49(0.091)$ & $0.00(0.000)$ & $0.51(0.091)$ \\
6 & 12 August 1992 & $0.88(0.249)$ & $0.30(0.069)$ & $0.08(0.040)$ & $0.62(0.076)$ \\
7 & 7 October 1992 & $1.54(0.238)$ & $0.57(0.065)$ & $0.02(0.017)$ & $0.41(0.065)$ \\
8 & 1 December 1992 & $0.67(0.185)$ & $0.51(0.056)$ & $0.03(0.020)$ & $0.45(0.056)$ \\
9 & 27 January 1993 & $0.65(0.472)$ & $0.83(0.065)$ & $0.00(0.000)$ & $0.17(0.065)$ \\
10 & 25 March 1993 & $\ldots$ & $0.66(0.113)$ & $0.04(0.046)$ & $0.29(0.111)$ \\
11 & 20 May 1993 & $\cdots$ & $0.43(0.139)$ & $0.14(0.099)$ & $0.43(0.141)$ \\
\hline
\end{tabular}

$\dagger$ Midpoint of five-day (periods 1-8) trapping period and first day of two-day (9-11) periods.

$\$$ Estimated from patch 1 capture history data using the approach of Pradel (1996).

$\S$ Estimated using reverse capture-recapture with multistate models.

portional reduction in the contribution from area 2 . Then we can predict the proportional reduction in $N_{i+1}^{1}$, and thus in $\lambda_{i}^{1}$, as $\alpha \gamma_{i+1}^{12}$.

As an aside, we note the analogy between the $\gamma_{i}^{r s}$ parameters and the quantity known as "derivation of the harvest"' in studies of harvested animal populations (e.g., Munro and Kimball 1982, Schwarz and Arnason 1990). Studies of derivation of the harvest attempt to estimate the relative contributions of various source populations to the animals harvested in a particular area. In such studies, efforts are made to estimate the probabilities that an animal harvested in the area of interest originated in each of a specified number of potential contributing source populations/areas. We believe that use of reverse-time, multistate, tag-recovery modeling deserves consideration as a possible means of estimating these probabilities more directly.

Estimation.-By analogy with the single-stratum situation, reverse-time capture-recapture analysis proceeds by using multistate estimation methods (e.g., Arnason 1972, 1973, Hestbeck et al. 1991, Brownie et al. 1993, Schwarz et al. 1993) on the reverse capture history. For example, a capture history for a three-sample study might be 102 . This animal was caught during sample period 1 at area 1 , not caught during sample period 2, and then caught at area 2 during sample period 3 . The reverse-time capture history for such an individual would be 201 . Recall that $\gamma_{i}^{r s}$ is the probability that an animal present in area $r$ during period $i$ was in area $s$ in period $i-1$. We can then model the reversetime history, conditional on capture in area 2 at time 3 , as $\gamma_{3}^{21}\left(1-p_{2}^{1}\right) \gamma_{2}^{11} p_{1}^{1}+\gamma_{3}^{22}\left(1-p_{2}^{2}\right) \gamma_{2}^{21} p_{1}^{1}$. The first term represents the probability that the individual was on area 1 in period 2, whereas the second term reflects the probability that the animal was on area 2 at time 2 . As the true location of the animal at period 2 is unknown, the probabilistic modeling must account for both possibilities. Note that the capture probabilities carry superscripts permitting the possibility of differences between the two study areas. As with the single-stratum models, standard software for multistate models (MSSURVIV [Hines 1994], MARK [White and Burnham 1999]) can be used to analyze reverse-time capture history data, and the usual survival-transition parameters (the $\phi_{i}^{r s}$ of Brownie et al. 1993) become the $\gamma_{i}^{r s}$.

Example analysis.-Here, we again use the data of the previous example, but we focus attention on one half of the grid. We divide the rectangular grid 4A into two square strata. Stratum 1 is defined by trapping rows 1-7 and stratum 2 is defined by trapping rows $9-15$. As the overall grid contains seven columns of traps, both strata are squares with $7 \times 7$ trapping stations. We again consider only adults and subadults (we will refer to these as adults) defined as animals of mass $>22$ g. The protocol for trapping and handling animals has been described (see One stage, one stratum: Example analysis; see the Supplementary Material for data).

For this example, we focus attention on stratum 1 and on the number of adults $\left(N_{i}^{1}\right)$ and the rate of increase in number of adults $\left(\lambda_{i}^{1}=N_{i+1}^{1} / N_{i}^{1}\right)$ in this stratum. We first used the model of Pradel (1996), with capturerecapture data only from stratum 1 (captures from row 8 or stratum 2 were simply entered as zero), to estimate population growth rates for this stratum. With some exceptions (e.g., sampling periods 4 and 5), the $\hat{\lambda}_{i}^{1}$ (Table 2) were not dissimilar from the estimated rates of increase for the entire grid, $\hat{\lambda}_{i}$ (Table 1).

We used reverse-time multistate modeling to estimate the relative contributions to population size and growth on stratum 1 from adults on stratum $1\left(\hat{\gamma}_{i}^{11}\right)$, adults on stratum $2\left(\hat{\gamma}_{i}^{12}\right)$, and new recruits (1$\left.\hat{\gamma}_{i}^{11}-\hat{\gamma}_{i}^{12}\right)$. Estimated contributions to stratum 1 of adults from stratum 2 were not large: $0.00 \leq \hat{\gamma}_{i}^{12} \leq$ 0.14 (Table 2). Consider the population change in stratum 1 between sampling periods 6 and 7. The estimated contributions from adults on stratum 1, adults on stratum 2 and new recruits were $0.57,0.02$, and 0.41 , respectively (Table 2 ). So a $5 \%$ change in adult survival on stratum 1 , or in the probability of an adult remaining on (not moving from) stratum 1, would have yielded 
a $2.85 \%$ (estimated $\mathrm{SE}=0.325 \%$ ) change in adult population growth on stratum 1 during the interval between periods 6 and 7. A 5\% change in adult survival on stratum 2 , or in the probability that an adult on stratum 2 would move to stratum 1 , would have produced a change of only $0.10 \%$ (estimated SE $=0.085 \%$ ) in $\lambda_{6}^{1}$. A $5 \%$ change in the number of recruits to stratum 1 would have produced a $2.05 \%$ (estimated SE $=0.325 \%)$ change in $\lambda_{6}^{1}$.

\section{Interest focused on the summed animals in multiple strata}

Demographic components.-Consider a population with no age structure, existing on a single study area. The population contains two types of individuals categorized by a threshold body mass, such that each animal's mass can be measured upon capture, and classification can be assigned as either heavy (stratum 1) or light (stratum 2). Animals are categorized at each capture period and can change state from one sampling period to another. An important difference between this situation and the previous one involving two locations is that, in the present case, we are interested in the entire population representing the sum of animals in the two states. We define the rate of increase of the population in the following manner:

$$
\lambda_{i}=\frac{N_{i+1}^{1}+N_{i+1}^{2}}{N_{i}^{1}+N_{i}^{2}}
$$

where the superscripts denote the two mass classes or states. The number of animals in the two classes in period $i+1$ can be written as

$$
\begin{aligned}
& N_{i+1}^{1}=S_{i}^{11}+S_{i}^{21}+B_{i}^{1} \\
& N_{i+1}^{2}=S_{i}^{12}+S_{i}^{22}+B_{i}^{2}
\end{aligned}
$$

where $S_{i}^{r s}$ denotes the number of animals in state $r$ at time $i$ that are alive and in state $s$ at time $i+1$, and $B_{i}^{s}$ denotes the number of new (not present at $i$ ) recruits to the population that are members of state $s$ at time $i$ +1 . The number of animals in each state at time $i+$ 1 thus represents the sum of (1) surviving animals from the same state in the previous time period, (2) surviving animals from the other state in the previous time period that made a transition between states, and (3) new recruits that were not members of the population the previous period.

We can model the numbers of animals in each of the three demographic components at time $i$ contributing to $N_{i+1}^{1}$ (Eq. 8) as one trinomial (the same trinomial as in Eq. 5) and the numbers of animals in each of the three components contributing to $N_{i+1}^{2}$ (Eq. 8) as a different trinomial:

$$
\begin{aligned}
P\left(S_{i}^{12}, S_{i}^{22}, B_{i}^{2} \mid N_{i+1}^{2}\right)= & \frac{\left(N_{i+1}^{2}\right) !}{\left(S_{i}^{12}\right) !\left(S_{i}^{22}\right) !\left(B_{i}^{2}\right) !}\left(\gamma_{i+1}^{21}\right)^{S_{i}^{12}} \\
& \times\left(\gamma_{i+1}^{22}\right)_{i}^{S_{i}^{22}}\left(1-\gamma_{i+1}^{21}-\gamma_{i+1}^{22}\right)^{B_{i}^{2}} .
\end{aligned}
$$

The $\gamma_{i}^{r s}$ are defined in a manner similar to the previous case as the probability that an animal in state $r$ during period $i$ was alive in state $s$ in period $i-1$.

Based on Expressions 5-9, decomposition of $E\left(\lambda_{i}\right)$ is accomplished as follows:

$$
\begin{aligned}
E\left(\lambda_{i}\right) \approx & {\left[E\left(S_{i}^{11}\right)+E\left(S_{i}^{21}\right)+E\left(B_{i}^{1}\right)+E\left(S_{i}^{12}\right)+E\left(S_{i}^{22}\right)\right.} \\
& \left.+E\left(B_{i}^{2}\right) 1\right]\left[E\left(N_{i}^{1}\right)+E\left(N_{i}^{2}\right)\right]^{-1} \\
= & {\left[\gamma_{i+1}^{11} N_{i+1}^{1}+\gamma_{i+1}^{12} N_{i+1}^{1}+\left(1-\gamma_{i+1}^{11}-\gamma_{i+1}^{12}\right) N_{i+1}^{1}\right.} \\
& \left.+\gamma_{i+1}^{21} N_{i+1}^{2}+\gamma_{i+1}^{22} N_{i+1}^{2}+\left(1-\gamma_{i+1}^{21}-\gamma_{i+1}^{22}\right) N_{i+1}^{2}\right] \\
& \times\left[E\left(N_{i}^{1}\right)+E\left(N_{i}^{2}\right)\right]^{-1} .
\end{aligned}
$$

In Expression 10, the members of each state of the population at time $i+1$ are classified using the $\gamma_{i+1}^{r s}$ into their respective components from the previous time period, $i$. So $\gamma_{i+1}^{11}, \gamma_{i+1}^{12}$, and $\left(1-\gamma_{i+1}^{11}-\gamma_{i+1}^{12}\right)$ reflect the relative contributions of the three components to $N_{i+1}^{1}$, and $\gamma_{i+1}^{21}, \gamma_{i+1}^{22}$, and $\left(1-\gamma_{i+1}^{21}-\gamma_{i+1}^{22}\right)$ represent the contributions to $N_{i+1}^{2}$. Because population growth is defined in terms of both states ( 1 and 2 ; Eq. 7 ), however, the relative influence of a demographic component on $\lambda_{i}$ now requires information not only on the $\gamma_{i+1}^{r s}$, but also on the relative composition of the population at time $i+1$ with respect to the two states. The relative state composition of the population at time $i+1$ is simply given by $N_{i+1}^{r} /\left(N_{i+1}^{1}+N_{i+1}^{2}\right)$, for $r=1$ or 2 .

Estimation.-As in the previous multistate application, we can use reverse-time multistate estimation methods (Brownie et al. 1993) to estimate the $\gamma_{i+1}^{r s}$, the probability that a member of state $r$ at time $i+1$ was in state $s$ in period $i$. The relative effect on population growth $\left(\lambda_{i}\right.$, Eq. 7) of a proportional reduction of $\alpha$ in one of the recruitment variables $\left(B_{i}^{r}\right)$ can be estimated as $\alpha\left(1-\hat{\gamma}_{i+1}^{r 1}-\hat{\gamma}_{i+1}^{r 2}\right)\left(\hat{N}_{i+1}^{r} /\left[\hat{N}_{i+1}^{1}+\hat{N}_{i+1}^{2}\right]\right)$, where the ratio, $\hat{N}_{i+1}^{r} /\left[\hat{N}_{i+1}^{1}+\hat{N}_{i+1}^{2}\right]$, estimates the proportion of animals in the population at time $i+1$ in state $r$. Note that one way of obtaining the state-specific abundance estimates needed for this estimation is to simply divide the number of animals captured in a state, $n_{i}^{r}$, by the corresponding estimated time- and state-specific capture probability $\left(\hat{N}_{i}^{r}=n_{i}^{r} / \hat{p}_{i}^{r}\right)$.

As another example, the relative effect on $\lambda_{i}$ of a proportional reduction of $\alpha$ in survival of animals in state 1 at time $i$ (survival of $N_{i}^{1}$; recall that some of these animals remain in state 1 and others move to state 2) can be estimated as

$$
\frac{\alpha\left(\hat{\gamma}_{i+1}^{11} \hat{N}_{i+1}^{1}+\hat{\gamma}_{i+1}^{21} \hat{N}_{i+1}^{2}\right)}{\hat{N}_{i+1}^{1}+\hat{N}_{i+1}^{2}} .
$$

This expression effectively weights the $\gamma_{i+1}^{r s}$ parameter estimates by the estimated proportional compositions of the population (at $i+1$ ) to which they pertain.

Estimation of the relative state composition of the population at $i+1 \mathrm{using} \hat{N}_{i+1}^{r} /\left[\hat{N}_{i+1}^{1}+\hat{N}_{i+1}^{2}\right]$ represents a very general approach that is appropriate even if the animals in the two states exhibit different capture prob- 
abilities. However, if animals in the two states exhibit similar capture probabilities, then this estimator is likely inefficient (e.g., see Skalski and Robson 1992) and can be replaced by $n_{i+1}^{r} /\left(n_{i+1}^{1}+n_{i+1}^{2}\right)$. The hypothesis of $p_{i+1}^{1}=p_{i+1}^{2}$ can be formally tested using a likelihood ratio test of competing multistate capture-recapture models (e.g., Brownie et al. 1993). The approach of conducting such a test and, when there is no evidence of different capture probabilities, using the estimator based on count statistics $\left(n_{i+1}^{r}\right)$, rather than the estimator based on estimated abundance $\left(\hat{N}_{i+1}^{r}\right)$, is similar to that recommended by Skalski and Robson (1992) and used by Nichols et al. (1994) in a closely related context.

\section{Two Ages, One Stratum}

Assume that we conduct a capture-recapture study at a single location and that animals can be classified upon capture as either young or adult. Further assume that the sampling takes place soon after reproduction (toward the end of the breeding season for seasonal breeders). We consider the situation where the length of time separating consecutive sample occasions corresponds to the time required for young animals to become adults. An example might be a population of ducks that we sample each year at the end of the breeding season. The sampling should be timed to follow the production of young sufficiently closely that the young animals caught represent young produced on (or very close to) the study area.

The sampling for this situation must follow the robust design of Pollock (1982) because of the need to estimate capture probability for young animals (see Discussion). This design includes primary sampling periods, between which the population is assumed to be open to gains and losses (the annual samples would be the primary samples in our duck example). Within each primary sample, there are two or more secondary sampling occasions. The secondary samples are typically spaced very close together in time in order to provide a good likelihood that the population is closed to gains and losses between secondary samples. In the duck example, we might catch birds on three consecutive days or perhaps two consecutive weeks, and in small mammal examples we might trap for five consecutive days.

\section{Demographic components}

Using superscripts to denote age class ( 2 = adult, 1 = young; e.g., the number of adults at $i+1$ is denoted as $N_{i+1}^{2}$ ), the finite rate of increase for the entire population (both age classes) can be written as:

$$
\lambda_{i}^{1+2}=\frac{N_{i+1}^{1}+N_{i+1}^{2}}{N_{i}^{1}+N_{i}^{2}}
$$

The finite rate of increase for the adult component of the population is given by the following:

$$
\lambda_{i}^{2}=\frac{N_{i+1}^{2}}{N_{i}^{2}} .
$$

For a population at stable age distribution, these two growth rates will be equal, $\lambda_{i}^{1+2}=\lambda_{i}^{2}$, but for a population exhibiting transient dynamics or experiencing temporal variation in vital rates, the two growth rates can be different.

The number of adults at time $i+1$ can be written as the sum of the three demographic components with respect to time $i$ :

$$
N_{i+1}^{2}=S_{i}^{22}+S_{i}^{12}+B_{i}^{2} .
$$

where $S_{i}^{22}$ is the number of adults present in the population at time $i$ that survive and are still present at time $i+1, S_{i}^{12}$ is the number of young animals present at time $i$ that survive to become adults at time $i+1$, and $B_{i}^{2}$ denotes the number of immigrants between $i$ and $i+1$ that are present as adults at time $i+1$.

As in the previous cases, we can view these components as random variables following a trinomial distribution conditional on $N_{i+1}^{2}$ and governed by parameters $\gamma_{i+1}^{2 s}$. These $\gamma_{i+1}^{2 s}$ parameters reflect the probability that an adult animal at time $i+1$ was in a particular demographic category (young, $s=1$; adult, $s=2$ ) at time $i$. The probability distribution of these different components contributing to the adult population at $i+$ 1 can be written as follows:

$$
\begin{aligned}
P\left(S_{i}^{12}, S_{i}^{22}, B_{i}^{2} \mid N_{i+1}^{2}\right)= & \frac{\left(N_{i+1}^{2}\right) !}{\left(S_{i}^{12}\right) !\left(S_{i}^{22}\right) !\left(B_{i}^{2}\right) !}\left(\gamma_{i+1}^{21}\right)^{S_{i}^{12}} \\
& \times\left(\gamma_{i+1}^{22}\right)^{S_{i}^{22}}\left(1-\gamma_{i+1}^{21}-\gamma_{i+1}^{22}\right)^{B_{i}^{2}} .
\end{aligned}
$$

In this two-age situation, we use the $\gamma_{i+1}^{2 s}$ to decompose the adult population at time $i+1\left(N_{i+1}^{2}\right)$ into relative contributions of adults at $i$, young at $i$, and immigrants. The corresponding decomposition for the growth rate of the adult component (defined in Eq. 12) is

$$
\begin{aligned}
E\left(\lambda_{i}^{2}\right) & \approx \frac{E\left(S_{i}^{12}\right)+E\left(S_{i}^{22}\right)+E\left(B_{i}^{2}\right)}{E\left(N_{i}^{2}\right)} \\
& =\frac{\gamma_{i+1}^{21} N_{i+1}^{2}+\gamma_{i+1}^{22} N_{i+1}^{2}+\left(1-\gamma_{i+1}^{21}-\gamma_{i+1}^{22}\right) N_{i+1}^{2}}{E\left(N_{i}^{2}\right)} .
\end{aligned}
$$

Assume an example in which $\gamma_{i+1}^{22}=0.7, \gamma_{i+1}^{21}=$ 0.2 , and $1-\gamma_{i+1}^{22}-\gamma_{i+1}^{21}=0.1$. Under this scenario, we would conclude that recruitment resulting from in situ reproduction made twice the contribution to adult population growth over the interval $i$ to $i+1$ as recruitment from immigration. Relative contributions of these two types of recruitment are relevant to characterizing habitats as sources or sinks (e.g., Pulliam 1988). These quantities can be used to draw inferences about the change in population growth of the adult component, $\lambda_{i}^{2}$, that would have resulted from a specified change in a demographic component at $i$. For example, a pro- 
portional reduction, $\alpha$, in young survival [i.e., new survival given by $(1-\alpha) \phi_{i}^{1}$ ] produces a proportional reduction in adult population growth of $\alpha \gamma_{i+1}^{21}$ [i.e., new adult growth rate can be obtained as $\left.\lambda_{i}^{2}\left(1-\alpha \gamma_{i+1}^{21}\right)\right]$.

If interest is focused on the growth rate of the entire population (young and adults), then we use Expression 12 for $\lambda_{i}^{1+2}$. Relative effects of changes in vital rates now involve the age composition of the population at $i+1$, as well as the relative contributions to the adult component at $i+1$, and the computations initially seem more complicated than for $\lambda_{i}^{2}$. However, in many sampling situations, the young animals in the population at $i+1$ can be viewed as the product of number of adults at $i+1$ and a per capita reproductive rate for adults. Thus, a proportional reduction of $\alpha \gamma_{i+1}^{21}$ in the adult population at $i+1$ produces an identical proportional reduction in the number of new young at $i+$ 1. The consequence of this reasoning is simply that $\alpha \gamma_{i+1}^{21}$ gives the proportional reduction in both $\lambda_{i}^{2}$ and $\lambda_{i}^{1+2}$ that would have resulted from proportional reduction $\alpha$ in young survival between $i$ and $i+1$.

It may be of interest to make inference about the influence of a reduction in reproductive rate, or one of its multiplicative components (e.g., probability of an adult breeding, clutch size, nest success), on population size or growth rate. For such inference, we would go directly to Eq. 11 and focus on $N_{i+1}^{1}$ Proportional reduction of magnitude $\alpha$ in reproductive rate, or one of its components, just prior to $i+1$, should lead to proportional reduction in $\lambda_{i}^{1+2}$ of $\alpha \hat{N}_{i+1}^{1} /\left(\hat{N}_{i+1}^{1}+\hat{N}_{i+1}^{2}\right)$. In the case of changes in reproductive rate, we would simply compute effects of such changes on $\lambda_{i}^{1+2}$ using the age-specific estimates of population size, with no need to resort to reverse-time estimation.

\section{Estimation}

As in the previous cases, we would like to use reverse-time capture-recapture to estimate relative contributions of different components at time $i$ to population growth between $i$ and $i+1$. However, the situation with multiple age classes is not as straightforward as in the previously described situations, where we simply reversed the time order of capture histories and applied the same models as used for standard time data. The standard models for estimating age-specific parameters (Pollock 1981, Lebreton et al. 1992) condition on animals released in the different age categories and model their capture histories separately.

For example, assume a two-age model (e.g., young and adult) and consider an animal caught in periods 1 and 3 but not in period 2. First assume that the animal was an adult (age, $r=2$ ) when released at period 1 , yielding capture history 202. Conditional on this release in period 1 , the probability associated with the remainder of the capture history is $\phi_{1}^{2}\left(1-p_{2}^{2}\right) \phi_{2}^{2} p_{3}^{2}$, where superscript again denotes age and where $\phi_{i}^{2}$ is the probability that an adult alive and in the population at time $i$ survives and is still in the population at $i+$
1. Now assume that a young animal (age, $r=1$ ) was released at time 1 . Conditional on this release, the corresponding probability for capture history 102 is: $\phi_{1}^{1}\left(1-p_{2}^{2}\right) \phi_{2}^{2} p_{3}^{2}$, where $\phi_{i}^{1}$ is the probability that a young animal at time $i$ survives and is an adult in the population at $i+1$. The only difference between the probabilities associated with these young and adult capture histories involves the initial survival probability that corresponds to the age of the released animal. After the interval between periods 1 and 2, the young animal becomes an adult and experiences the same survival and capture probabilities as the animal marked as an adult.

When we reverse the time order of the capture history data, it is clear that we cannot simply use the standard multi-age capture-recapture models. In the two-age case, we will be conditioning on animals that are all adults at time $i+1$ and then asking what proportion of these animals were adults at time $i$, young at time $i$, or immigrants entering between $i$ and $i+1$ (see Eqs. 13 and 14). So, instead of conditioning on animals in two distinct age classes and estimating their respective probabilities of appearing in a single age class in the next time period, we are conditioning on animals in one age class (adults) and estimating their probabilities of having been in one of three different classes (young, adult, immigrant) in the previous time period. The reverse-time approach to multi-age modeling thus requires multistate modeling.

Conditional on capture of an adult at period 3, we would model reverse history 202 as $\gamma_{3}^{22}\left(1-p_{2}^{2}\right) \gamma_{2}^{22} p_{1}^{2}$, where the $\gamma_{i}^{22}$ parameters reflect the probability that an adult animal alive in period $i$ was an adult in the population at time $i-1$. Reverse capture history 201, again conditional on release of an adult in period 3 , can be modeled as $\gamma_{3}^{22}\left(1-p_{2}^{2}\right) \gamma_{2}^{21} p_{1}^{1}$, where $\gamma_{i}^{21}$ denotes the probability that an adult at time $i$ was a young animal at time $i-1$. Note that unlike the previous modeling with multistate models, the probability structure associated with a " 0 " in the capture history is modeled as a single path rather than as the sum of alternative possible pathways. This is because an animal that is young in period 1 cannot also have been young at period 2 , because of the correspondence of the interval between sample periods and the interval required to make an age transition (an animal grows deterministically from young to adult in a single interval).

The other aspect of the probability modeling that differs between standard and reverse-time parameterizations is the need for capture probabilities for young animals $\left(p_{i}^{1}\right)$ with the reverse-time approach. Under standard time, animals are released as young, but survivors to the next sampling period are adults and are thus recaptured with probability $p_{i}^{2}$. However, estimation of $\gamma_{i}^{21}$ under the reverse-time approach requires information about $p_{i-1}^{1}$. This requirement explains the need for the robust design when using reverse-time modeling with data from multiple age classes. Capture 
TABLE 3. Definitions of unknown random variables that require estimation and statistics that result from a capturerecapture study of a population with young (state, $r=1$ ) and adult (state, $r=2$ ) age classes.

\begin{tabular}{|c|c|}
\hline Symbol & Definition \\
\hline \multicolumn{2}{|l|}{ Statistics } \\
\hline$n_{i}^{l}$ & $\begin{array}{l}\text { Total no. young animals caught in primary pe- } \\
\text { riod } i \text {. }\end{array}$ \\
\hline$n_{i}^{2}$ & $\begin{array}{l}\text { Total no. adult animals caught in primary peri- } \\
\quad \text { od } i \text {. }\end{array}$ \\
\hline$m_{i, i-1}^{21}$ & $\begin{array}{l}\text { Members of } n_{i}^{2} \text { that were caught as young ani- } \\
\text { mals in primary period } i-1 \text {. }\end{array}$ \\
\hline$m_{i, i-1}^{22}$ & $\begin{array}{l}\text { Members of } n_{i}^{2} \text { that were caught as adult ani- } \\
\text { mals in primary period } i-1 \text {. }\end{array}$ \\
\hline \multicolumn{2}{|c|}{ Unknown random variables that require estimation } \\
\hline$M_{i, i-1}^{21}$ & $\begin{array}{l}\text { Members of } n_{i}^{2} \text { that were in the sampled area as } \\
\text { young animals in primary period } i-1 \text { (but } \\
\text { not necessarily captured in } i-1 \text { ). }\end{array}$ \\
\hline$M_{i, i-1}^{22}$ & $\begin{array}{l}\text { Members of } n_{i}^{2} \text { that were in the sampled area as } \\
\text { adult animals in primary period } i-1 \text { (but } \\
\text { not necessarily captured in } i-1 \text { ). }\end{array}$ \\
\hline
\end{tabular}

probability for young animals cannot be estimated from standard age-specific capture-recapture models for open populations. Instead, captures and recaptures of young from secondary samples under a robust design approach are needed in order to estimate capture probability for young (also see Nichols and Pollock 1990).

Estimation using multistate models with the robust design can be carried out in either of two ways. First, we can build a joint likelihood (e.g., Kendall et al. 1995, 1997; Schwarz and Stobo 1997) with separate components for modeling the capture-recapture data over secondary and primary periods. This approach is appropriate when the models being used for the secondary sample data are those for which direct maximum likelihood estimation of model parameters is straightforward (e.g., closed models involving behavioral response and time are in this category).

When direct maximum likelihood estimation under a closed model is not so straightforward (e.g., as in estimators for abundance and average capture probability under models that include heterogeneity of capture probability such as model $\mathrm{M}_{\mathrm{h}}$; Burnham and $\mathrm{Ov}$ erton 1978), then we can follow a second possible approach to estimation of the $\gamma_{i}^{r s}$ parameters. Table 3 lists definitions of the statistics that result from a capturerecapture study of a population with young and adult age classes. Table 3 further delineates two unknown random variables requiring estimation.

Estimation of the $\gamma_{i}^{r s}$ requires estimation of the $M_{i, i-1}^{r s}$, and this can be done using either of two approaches. One straightforward approach is to simply condition on the $m_{i, i-1}^{r s}$ (all of these animals were caught in both time periods, $i$ and $i-1$ ). The capture histories of these animals over the secondary trapping periods of primary period $i-1$ can be used to estimate $M_{i, i-1}^{r s}$ using the jackknife estimator for $\mathrm{M}_{\mathrm{h}}$ proposed by Burnham and Overton (1978), or any other estimator appropriate for the secondary period data (e.g., Otis et al. 1978, Rexstad and Burnham 1991, Lee and Chao 1994). In this manner we obtain an estimate of "population size" for a subset of the animals captured in state $s$ at $i-1$; those that were also captured at $i$ in state $r$. So for estimation of $M_{i, i-1}^{21}$, we would condition on all the animals caught as adults (state 2) at time $i$ and as young (state 1 ) at time $i-1$ (the $m_{i, i-1}^{21}$ ). Use of the capture histories of these animals over the secondary periods of primary period $i-1$ would be used with a capture-recapture estimator (typically from a closed model), and the resulting abundance estimate would correspond to $\hat{M}_{i, i-1}^{21}$.

The other approach to estimation of $M_{i, i-1}^{r s}$ is to focus on all animals of state $s$ caught at $i-1$, regardless of whether they were also caught in state $r$ at time $i$ (we have denoted these animals as $n_{i-1}^{s}$ ). Use of the capture histories of these animals over the secondary periods of primary period $i-1$ with a closed model estimator yields an abundance estimate for members of that state in period $i-1\left(\hat{N}_{i-1}^{s}\right)$. The average probability that an animal in state $s$ at primary period $i-1$ is caught at least once during $i-1$ (we denote this probability as $\left.\bar{p}_{i-1}^{s}\right)$ is then estimated in the following manner:

$$
\hat{\bar{p}}_{i-1}^{s}=\frac{n_{i-1}^{s}}{\hat{N}_{i-1}^{s}} .
$$

We can then estimate $M_{i, i-1}^{r s}$ as

$$
\hat{M}_{i, i-1}^{r s}=\frac{m_{i, i-1}^{r s}}{\hat{\bar{p}}_{i-1}^{s}} .
$$

This latter estimator should be more efficient than the former estimator that was based on the subset of animals caught at $i-1$ in state $s$ that were also caught at $i$ in state $r$.

Estimates of the $M_{i, i-1}^{r s}$ can then be used to estimate $\gamma_{i}^{r s}$. In the two-age problem presented here, we estimate the two relevant parameters, $\gamma_{i}^{21}$ and $\gamma_{i}^{22}$, as follows:

$$
\hat{\gamma}_{i}^{2 s}=\frac{\hat{M}_{i, i-1}^{2 s}}{n_{i}^{2}} \quad s=1,2 .
$$

Use of reverse-time, two-age, capture-recapture data obtained under a robust design can thus involve either joint multistate likelihoods or estimators such as that in Eq. 18 to directly estimate the $\gamma_{i}^{2 s}$.

\section{Example analysis}

The data for this example again involve meadow voles trapped at Patuxent Wildlife Research Center, this time during the summer and fall of 1981 (Nichols et al. 1984a). This older data set used the robust design with primary sampling conducted at six monthly periods. This interval between sampling periods was more likely than our recent sampling schedule to meet the assumptions underlying our estimation approach for separating recruitment resulting from in situ reproduction and from immigration (see Nichols and Pollock 1990, Yoccoz et al. 1993). The grid was a $10 \times 10$ 
TABLE 4. Estimates of population change for both adults $\left(\hat{\lambda}_{i}^{2}\right)$ and adults + young $\left(\hat{\Lambda}_{i}^{1+2}\right)$, and relative contributions to adult population growth of adult survival $\left(\hat{\gamma}_{i}^{22}\right)$, fecundity and young survival $\left(\hat{\gamma}_{i}^{21}\right)$, and immigration from outside the study area $\left(1-\hat{\gamma}_{i}^{22}-\hat{\gamma}_{i}^{21}\right)$ for meadow voles trapped at Patuxent Wildlife Research Center, Laurel, Maryland, June-December, 1981.

\begin{tabular}{|c|c|c|c|c|c|c|}
\hline $\begin{array}{c}\text { Trapping } \\
\text { period }\end{array}$ & Date $\dagger$ & $\hat{\lambda}_{\mathrm{i}}^{2}\left(\operatorname{SE}\left[\hat{\lambda}_{\mathrm{i}}^{2}\right]\right) \ddagger$ & $\begin{array}{c}\hat{\Lambda}_{i+2}^{1+2} \\
\left(\operatorname{SE}\left[\hat{\lambda}_{i}^{1+2}\right]\right) \ddagger\end{array}$ & $\hat{\gamma}_{i}^{22}\left(\operatorname{sE}\left[\hat{\gamma}_{i}^{22}\right]\right) \S$ & $\hat{\gamma}_{i}^{21}\left(\operatorname{sE}\left[\hat{\gamma}_{i}^{21}\right]\right) \S$ & $\begin{array}{l}1-\hat{\gamma}_{i}^{22}-\hat{\gamma}_{i}^{21}(\mathrm{SE} \\
\left.\left[1-\hat{\gamma}_{i}^{22}-\hat{\gamma}_{i}^{21}\right]\right) \S\end{array}$ \\
\hline 1 & 29 June 1981 & $1.24(0.039)$ & $1.20(0.052)$ & & $\cdots$ & \\
\hline 2 & 3 August 1981 & $0.81(0.036)$ & $0.80(0.045)$ & $0.68(0.042)$ & $0.11(0.051)$ & $0.22(0.058)$ \\
\hline 3 & 31 August 1981 & $0.97(0.041)$ & $1.24(0.066)$ & $0.74(0.045)$ & $0.03(0.025)$ & $0.23(0.047)$ \\
\hline 4 & 5 October 1981 & $0.97(0.030)$ & $1.00(0.048)$ & $0.74(0.046)$ & $0.02(0.018)$ & $0.25(0.047)$ \\
\hline 5 & 2 November 1981 & $1.51(0.041)$ & $1.13(0.044)$ & $0.60(0.049)$ & $0.25(0.056)$ & $0.15(0.052)$ \\
\hline 6 & 6 December 1981 & & $\ldots$ & $0.62(0.040)$ & $0.22(0.044)$ & $0.16(0.041)$ \\
\hline
\end{tabular}

$\dagger$ Midpoint of five-day trapping period.

\$ Estimated as ratios of Lincoln-Petersen abundance estimates.

$\S$ Estimated using reverse capture-recapture with multistate models under the robust design.

square of trapping stations with $7.6 \mathrm{~m}$ trap spacing. This study used Fitch traps (Rose 1973) baited with whole corn and containing hay. The protocol for marking and handling animals was the same as described for the more recent study. Adults were defined as voles $>22 \mathrm{~g}$, and animals of smaller mass were designated as young. The data and analysis are again provided in the Supplementary Material.

As noted in the description of the approach to estimation with two ages, we required a robust design with multistate modeling. We first developed a single likelihood containing both closed and open components (Kendall et al. 1995, 1997). For the modeling of closed data, we used a Lincoln-Petersen approach considering the first two days of trapping as "period 1" and the second three days as "period 2" (see Menkins and Anderson 1988). One of these period-specific capture probability parameters was then rewritten as a function of the overall probability of being caught in at least one of the two periods (see Kendall et al. 1995, 1997), as it is this latter parameter that corresponds to the open-model portion of the likelihood. Closed-model data (obtained over secondary periods) were stratified by age (young, adult) in order to estimate capture probability for each age class. The young data were adequate to estimate capture probability well for primary periods 4 and 5 , but not for periods $1-3$. So the secondary capture-recapture data for young voles in periods $1-3$ were modeled with the same parameters (assumed to be constant over the three primary periods) in order to permit estimation. The open-model portion of the likelihood was based on a multistate model. Because we used a reverse-time approach, certain transition probabilities were not possible and set equal to zero (a young animal at $i$ could not have existed at time $i-1$, so $\gamma_{i}^{11}=\gamma_{i}^{12}=0$ ).

Population sizes were estimated using Lincoln-Petersen estimates of abundance by age (e.g., Seber 1982, Menkins and Anderson 1988). We used these population size estimates to estimate two different rates of increase, one corresponding to only the adult component of the population $\left(\lambda_{i}^{2}\right)$ and the other corresponding to the sum of adults and young $\left(\lambda_{i}^{1+2}\right)$.
The multistate model for the robust design approach was implemented using program SURVIV (White 1983) coded in a manner similar to that used in MSSURVIV (Hines 1994). The fit of the vole data to the model was judged to be acceptable. Estimated rates of increase ranged $0.81-1.51$ for adults and $0.80-1.24$ for adults and young combined (Table 4 ). The reverseorder modeling indicated that surviving adults were the largest contributors to population growth rate, constituting $0.60-0.74$ of the adult population throughout the study (values of $\hat{\gamma}_{i}^{22}$ in Table 4). The proportion of adults that were young on the study area in the previous period was estimated to range $0.02-0.25$ (values of $\hat{\gamma}_{i}^{21}$ in Table $4)$. The estimated proportion of adults that were new immigrants ranged $0.15-0.25$ (values of $\left[1-\hat{\gamma}_{i}^{22}-\right.$ $\left.\hat{\gamma}_{i}^{21}\right]$ in Table 4). Recruitment of adults was dominated by immigrants in some periods ( 3 and 4 ) and by surviving young in others (5 and 6), indicating substantial temporal variation in the mechanisms responsible for population growth. Knowledge of such changes in the source of new recruits to a population is important to understanding animal population dynamics and regulation and to managing animal populations.

\section{DISCUSSION}

\section{Reverse-time capture-recapture methods}

The methods that we have presented permit retrospective looks at population growth and assignment of proportional contributions of different demographic components to this growth. For several sampling situations that differ with respect to number of ages and strata (with respect to either location or animal characteristics) sampled, we have presented methods for estimating $\gamma_{i+1}$ parameters using reverse-time capturerecapture. These parameters reflect the probability that an animal in a population (or a component of a population) at time $i+1$ was a member of some sampled group at time $i$ or was instead a new recruit (not a member of one of the sampled groups). Depending on the sampling situation and the population growth rate that is of primary interest (e.g., that of adults or that of both young and adults), the estimated $\gamma_{i+1}$ parameters 
either represent the proportional contributions of the different components to population growth, or else can be used to compute these contributions.

As noted, traditional CJS modeling (Cormack 1964, Jolly 1965, Seber 1965) of capture-recapture data conditions on numbers of animals released and models the subsequent capture history with survival and capture probability parameters, $\phi_{i}$ and $p_{i}$, respectively. Our reverse-time approach conditions on final captures and models with $\gamma_{i}$ and $p_{i}$ parameters. However, the usual Jolly-Seber (Jolly 1965, Seber 1965) approach to capture-recapture modeling also permits estimation of the unknown random variables, $N_{i}=$ population size at time $i$, and $B_{i}=$ no. new animals entering the population between times $i$ and $i+1$ and present in the population at $i+1$. For those more familiar with standard Jolly-Seber modeling, we provide the following relationships for the single-age, single-location sampling situation:

$$
\begin{gathered}
\gamma_{i+1}=E\left(\frac{N_{i} \phi_{i}}{N_{i+1}}\right)=1-E\left(\frac{B_{i}}{N_{i+1}}\right) \\
E\left(\lambda_{i}\right)=E\left(\frac{N_{i+1}}{N_{i}}\right)=\phi_{i}+E\left(\frac{B_{i}}{N_{i}}\right) .
\end{gathered}
$$

As shown by Pradel (1996), we can also write $\lambda_{i}$ as a function of $\phi_{i}$ and $\gamma_{i}$. This can be seen by first writing in two different ways the expected number of animals alive in two consecutive sampling periods, $i$ and $i+1$ :

$$
E\left(N_{i} \phi_{i}\right)=E\left(N_{i+1} \gamma_{i+1}\right) .
$$

Rearrangement of Eq. 20 yields the following equality:

$$
E\left(\lambda_{i}\right)=\frac{\phi_{i}}{\gamma_{i+1}}
$$

The Jolly-Seber estimators, $\hat{N}_{i}$ and $\hat{B}_{i}$, are known to be less robust to deviations from the underlying model assumption of homogeneous capture probabilities than estimators for other parameters such as $\hat{\phi}_{i}$ (e.g., see Carothers 1973, Pollock 1982, Pollock et al. 1990). The relationships shown in Eq. 19 show that both $\lambda_{i}$ and $\gamma_{i}$ can be written as functions of $\hat{N}_{i}$ and $\hat{B}_{i}$, and this should provide cause for concern about the robustness of the reverse-time $\hat{\gamma}_{i}$ and the $\hat{\lambda}_{i}$ of Pradel (1996). For example, $\hat{\phi}_{i}$ is not biased by permanent trap response in capture probabilities (inducing different capture probabilities for marked and unmarked animals), because the estimator is based on application of the capture probability estimates to marked animals only (Nichols et al. 1984b). However, the reverse-time estimator, $\hat{\gamma}_{i}$, involves application of estimated (based on marked animals only) capture probabilities to both marked and unmarked animals, and should thus be biased by permanent trap response behavior (e.g., also see Franklin et al. [1999]). Until additional work is done on these estimators, it seems wise to view them as being similar to the Jolly-Seber $\hat{N}_{i}$ and $\hat{B}_{i}$ with respect to robustness. In particular, the assumption of equal capture probabilities for marked and unmarked animals will be important to these estimators, in contrast to their lack of importance for $\hat{\phi}_{i}$, and should thus receive consideration in designs of studies that will involve these estimators.

\section{Related concepts and methods}

Key factor analysis and sensitivity/elasticity analyses are methods used to address questions similar to those for which our $\gamma_{i}$ can be used. Key factor analysis (e.g., Morris 1959, Varley and Gradwell 1960) and related approaches (e.g., Reynolds and Sauer 1991, Johnson et al. 1992) are based on time series of estimates of population size or growth rate. Morris (1959:580) defined key factors as those "that cause a variable... mortality and appear to be largely responsible for the observed changes in population size." Correlation and regression analyses are used to investigate the relative correspondence between temporal variation in the different vital rates and in population size or growth. Such analyses are used to draw inferences about which vital rates are most closely associated with temporal variation in population growth. Key factor analysis and related approaches have seen only limited success in animal population ecology. An important reason for this is the failure to properly incorporate sampling variation and covariation in applications of the key factor approach (Kuno 1971, Manly 1977, 1979).

Like key factor analysis, our approach to estimating $\gamma_{i}$ is retrospective in nature. However, our approach does not share the key factor focus on temporal covariation of population change and demographic components or vital rates. Instead, we present a direct decomposition of population growth rate into demographic components. Our $\gamma_{i}$ parameters are not based on temporal covariation with population growth, but instead reflect the magnitudes of contributions to growth.

An alternative approach for considering the relative influences of the different vital rates on population growth is to focus on the sensitivity of the asymptotic rate of increase to changes in the vital rates. This approach is not based on observed temporal variation and covariation, but instead uses population projection models (e.g., Bernardelli 1941, Lewis 1942, Leslie 1945, Caswell 1989) and focuses on potential changes in vital rates and corresponding effects on the asymptotic rate of increase (the rate of increase that would apply if the population were exposed to the same set of vital rates repeatedly). Numerical investigations were initially used in following this general approach (e.g., Lewontin 1965, Leslie 1966, Mertz 1971), and, more recently, analytic expressions have been developed for investigating sensitivity (Caswell 1978, 1989) and elasticity (Caswell et al. 1984, de Kroon et al. 1986, van Groenendael et al. 1988, Caswell 1989). 
Sensitivity analyses compute effects on rate of increase of absolute changes in vital rates, whereas elasticity analyses compute effects on rate of increase of proportional changes in vital rates (Caswell 1989). For example, if $a_{i j}$ is an element (a vital rate or a function of such rates) of a projection matrix that defines an asymptotic rate of increase of $\lambda$, then the sensitivity $\left(s_{i j}\right)$ of $\lambda$ to changes in $a_{i j}$ is defined by (e.g., Caswell 1989) as follows:

$$
s_{i j}=\frac{\partial \lambda}{\partial a_{i j}} .
$$

Relative sensitivity or elasticity $\left(e_{i j}\right)$ is then defined as follows (e.g., Caswell 1989):

$$
e_{i j}=\frac{\partial \log \lambda}{\partial \log a_{i j}}=\frac{\partial \lambda}{\partial a_{i j}} \frac{a_{i j}}{\lambda} .
$$

The $\gamma_{i+1}$ parameters estimated using our approach are closely related to the concept of elasticity of $\lambda_{i}$ with respect to demographic components and their associated vital rates. Consider our first example in which we decomposed expected population growth rate, $E\left(\lambda_{i}\right)$, into components associated with survivors from the previous period, $E\left(S_{i}\right)$, and new recruits, $E\left(B_{i}\right)$ (Eq. 3). We can compute a time-specific analog of elasticity for the survivor component as

$$
\frac{\partial \log E\left(\lambda_{i}\right)}{\partial \log E\left(S_{i}\right)}=\gamma_{i+1} .
$$

If we prefer to focus on vital rates associated with demographic components rather than the components themselves, then we can express the numbers of survivors and recruits as functions of the population at time $i$ and again compute an analog of elasticity. If we denote survival rate from time $i$ to $i+1$ as $E\left(S_{i} / N_{i}\right)=$ $\phi_{i}$, then the analog of elasticity of $E\left(\lambda_{i}\right)$ with respect to $\phi_{i}$ is given by

$$
\frac{\partial \log E\left(\lambda_{i}\right)}{\partial \log \phi_{i}}=\gamma_{i+1} .
$$

Although these proportional contribution parameters $\left(\gamma_{i}\right)$ are, in a sense, analogous to elasticities derived from population projection matrices, these quantities differ in several respects. Perhaps the most obvious difference involves the asymptotic nature of elasticity measures derived from projection matrices, contrasted with the applicability of the $\gamma_{i}$ parameters to specific time intervals $(i-1$ to $i$ ). So we cannot comfortably use a specific $\hat{\gamma}_{i}$ to characterize a population over a long period of time (although a mean of $\hat{\gamma}_{i}$ might be useful for such a purpose), and neither can we expect an asymptotic elasticity value to necessarily be a useful descriptor for population change over a specific interval. The asymptotic nature of sensitivity and elasticity analyses leads to uncertainty about their relevance to situations involving either transient dynamics that precede asymptotic behavior, or simple temporal variation in vital rates and population growth. With respect to temporal variation in vital rates, we might expect sensitivity and elasticity analyses to provide reasonable approximations for situations involving relatively small temporal variation, but perhaps not for populations inhabiting highly variable environments. Generally, we would expect the $\gamma_{i}$ parameters to be more useful in retrospective analyses, and true elasticities to be more useful for prospective analyses.

Another difference between elasticities and $\gamma_{i}$ involves geographic closure and the incorporation of movement into inferences about population change. Projection matrix approaches are ideal for populations that are geographically closed. As they are typically parameterized with birth and death rates, they are excellent for studying evolutionary questions (movement is not a fitness component and is only relevant to fitness to the extent that it influences birth or death rates). If our attention is focused on changes in numbers of animals inhabiting a particular area of interest, however, movement is likely to be a very important contributor to population dynamics. We can estimate $\gamma_{i}$ parameters corresponding to contributions from other sampled locations and, in some cases, from all nonsampled areas (e.g., the example with two ages and one stratum). Thus, inferences about the relative contributions of different demographic components to actual populations in ecological time are likely to be more easily addressed using the reverse-time capture-recapture approach. In particular, the ability to make statements about the relative contributions of, for example, in situ reproduction and immigration to population growth (e.g., Connor et al. 1983, Nichols and Pollock 1990) seems very relevant to questions about source-sink (Pulliam 1988) and openrecruitment (Roughgarden et al. 1985, 1988) systems.

Single-location population projection matrices typically reflect an asymmetry with respect to movement. The complements of survival rate estimates computed using capture-recapture and several other methods include both mortality and permanent emigration from the study area. Such survival estimates are often combined in projection matrices with fecundity estimates that are based solely on components of reproductive rate (e.g., litter size, clutch size, brood size at fledging). Matrices constructed using such estimates thus include movement in the complements of survival rates (the components of loss), but not in the fecundity parameters (the components of gain). One consequence of the movement asymmetry is that asymptotic rates of population increase computed from such matrices are frequently too small. Another consequence is an inability to draw inferences about the relative contribution of immigration to population growth.

Questions involving asymptotic rate of increase could be addressed using multistratum projection models (e.g., Rogers 1966, Schoen 1988, Lebreton 1996) incorporating movement. However, use of this approach to deal with movement requires that at least one of the modeled 
strata represent "the rest of the world" or all potential sources of immigrants other than the locations under detailed study. Although this is conceptually possible, the modeling of the dynamics of such "catch-all" strata is likely to be very difficult because of lack of information, yet very important to asymptotic characteristics of the metapopulation system.

A minor methodological point regarding the comparison of the $\gamma_{i}$ and sensitivites/elasticities involves statistical inference. Resampling approaches, such as the jackknife and bootstrap, can be used for inference on any demographic statistic computed from projection matrices (Caswell 1989), including sensitivity or elasticity values. The approach described in this paper permits direct estimation of sampling variances and covariances associated with the $\gamma_{\iota}$ parameters reflecting relative contributions to population growth.

Although we have focused on the relationship between the $\gamma_{i}$ and elasticity, we note additional relationships between $\gamma_{i}$ and other quantities derived from projection matrix asymptotics. The stable age- (or stage-) vector provides the asymptotic proportion of the population in each class or stage of the population vector (e.g., Caswell 1989). In the case of a standard age-structured Leslie (1945) matrix, for example, $w_{i}$ represents the asymptotic proportion of the population in age class $i$. Because of the deterministic nature of age transitions, a member of class $w_{i}$ in one year was necessarily a member of $w_{i-1}$ the previous year. So the stable-age vector specifies the relative contributions of different age classes in the previous year to the current year's population. Estimates of $\gamma_{i}$ are especially useful when members of age or stage class $i$ at time $t$ are derived from two or more ages, stages, or locations at time $t-1$.

Finally, we note that the thinking underlying Fisher's reproductive value (e.g., Fisher 1930, Caswell 1989) is not dissimilar to that which underlies our view of the $\gamma_{i}$. Reproductive value for an individual of age $i$, $v_{i}$, is often defined as the value of such an individual to future population growth, expressed relative to the values of individuals of different ages. Perhaps another reasonable perspective results from viewing some ultimate population (a population viewed at some time in the distant future) and asking the question: "What fraction of the individuals in this future population are descendants of each of the different age classes at some time in the past (the time at which reproductive value is computed)?" In this sense, reproductive value can be viewed as a reverse-time look from some future population, asking about the proportional contributions to it of the different components of a specified past population. It might be possible to compute analogs of Fisher's reproductive value using $\hat{\gamma}_{i}$ in conjunction with estimated fecundity or reproductive rates to compute proportions of animals at some time $k$ that are survivors or descendants of animals in particular classes at some previous time $j$.
Uses of $\hat{\gamma}_{i}$

Direct estimation of the $\gamma_{i}$ parameters permits assessment of temporal variation in the relative contributions of different demographic components, a topic of recent interest in population dynamics (Gaillard et al. 1998). Maximum likelihood estimation of the $\gamma_{i}$ as in our examples yields direct estimates of sampling variances and covariances. Even with use of heterogeneity models requiring jackknife estimators of the $\gamma_{i}$ (e.g., the two-age, one-stratum example), variances can be estimated using the bootstrap or other approaches. Estimates of $\gamma_{i}$ and associated sampling variances and covariances over a period of time can then be used to estimate true temporal variance in the relative contribution of a component of interest using a variance components approach (see Burnham et al. 1987, Skalski and Robson 1992, Link and Nichols 1994, Gould and Nichols 1998, White and Burnham 1999). The relative variability vs. stability of a population with respect to the contributions of different demographic components to population growth would seem to be an interesting characteristic relevant to predictions and projections of future population dynamics.

The likelihood framework can also be used to assess the utility of reduced-parameter models incorporating restrictive assumptions about temporal variation in the proportional contribution parameters. For example, we might consider one model in which the relative contribution of a particular demographic component varies over time $\left(\gamma_{i}\right.$, where the $i$ denotes time) and another where the component is modeled as a constant $\left(\gamma_{i}=\gamma\right)$. The likelihood ratio test between these two models and their relative Akaike's Information Criterion (AIC) values (Burnham and Anderson 1998) provide information about temporal variation in $\gamma_{i}$. In particular, evaluation of the assumption that $\gamma_{i}=\gamma$ should be relevant to the question of whether use of asymptotic elasticities derived from projection matrices is reasonable.

In some instances, it may be useful to model the $\gamma_{i}$ using an ultrastructural modeling approach (e.g., Lebreton et al. 1992). We might consider modeling the $\gamma_{i}$ as a function of environmental covariates or information about neighboring populations. In our meadow vole example, we might predict an increase in the $\gamma_{i}$ associated with immigration from areas surrounding the sampled grid(s) during periods when surrounding habitat experienced dramatic modification (e.g., mowing). Similarly, environmental variables thought to be associated with movement, reproduction, or survival could be used to model the appropriate $\gamma_{i}$.

This paper has focused on the possible relevance of quantities (the $\gamma_{i}$ ) that can be directly estimated from capture-recapture data to quantities (elasticities) based on deterministic theory for discrete-time populations. However, the estimated $\gamma_{i}$ do not require any restrictive assumptions about absence of temporal variation in vital rates and may thus be useful in the context of sto- 
chastic population models as well. For example, Tuljapurkar (1990) has presented a stochastic analog of Caswell's (1978) general sensitivity expression appropriate for stationary stochastic processes, and we might expect a close relationship between this expression and the estimated $\gamma_{i}$ in the presence of stationarity. If the demographic process is not stationary, then estimates of $\gamma_{i}$ (and estimates of their temporal variance) should still provide useful information about the relative contributions of demographic components to stochastic population growth.

In conclusion, we hope that the methods presented in this manuscript will be useful in the demographic analysis of animal populations. We view these estimation methods simply as contributions to the animal ecologist's toolbox, which already includes standard capture-recapture methods for estimation of abundance and vital rates, and projection matrix methods for investigating asymptotic characteristics of populations governed by specified sets of vital rates. We also view these methods, together with the methods of Pradel (1996) for direct estimation of $\lambda$ from capture-recapture data, as providing a step toward the unification of distinct approaches to the study of animal population dynamics. Demographic estimation and projection are closely related endeavors, and it is important to recognize their similarities as well as their differences. It is perhaps useful to think of demographic projection methods as more closely tied to the theory of population dynamics. If this perspective is reasonable, then it is certainly important to test predictions deduced from theory, and inference methods such as those presented here should be useful for that purpose.

\section{ACKNOWLEDGMENTS}

This work was conducted under the U.S.-France Cooperative Science Program as part of the project "Modeling and Estimating Recruitment Dispersal in Animal Populations.' The project was supported by the Centre National de la Recherche Scientifique (Action CNRS/NSF 3879), the National Science Foundation, Division of International Programs (grant number INT-9603507), and by the Biological Resources Division of the United States Geological Survey. An anonymous referee provided very useful comments on an earlier draft of this manuscript, as did Evan Cooch, Bill Kendall, Mark Lindberg, and Scott Mills. Data for the first two examples came from a field project led by Rob Hinz.

\section{Literature Cited}

Arnason, A. N. 1972. Parameter estimates from mark-recapture experiments on two populations subject to migration and death. Researches on Population Ecology 13:97-113.

Arnason, A. N. 1973. The estimation of population size, migration rates and survival in a stratified population. Researches on Population Ecology 15:1-8.

Bernardelli, H. 1941. Population waves. Journal of the Burma Research Society 31:1-18.

Brownie, C., J. E. Hines, J. D. Nichols, K. H. Pollock, and J. B. Hestbeck. 1993. Capture-recapture studies for multiple strata including non-Markovian transition probabilities. Biometrics 49:1173-1187.

Burnham, K. P., and D. R. Anderson. 1998. Model selection and inference. Springer-Verlag, New York, New York, USA.
Burnham, K. P., D. R. Anderson, G. C. White, C. Brownie, and K. H. Pollock. 1987. Design and analysis methods for fish survival experiments based on release-recapture. American Fisheries Society Monograph 5.

Burnham, K. P., and W. S. Overton. 1978. Estimation of the size of a closed population when capture probabilities vary among animals. Biometrika 65:625-633.

Carothers, A. D. 1973. The effect of unequal catchability on Jolly-Seber estimates. Biometrics 29:79-100.

Caswell, H. 1978. A general formula for the sensitivity of population growth rate to changes in life history parameters. Theoretical Population Biology 14:215-230.

Caswell, H. 1989. Matrix population models. Sinauer Associates, Sunderland, Massachusetts, USA.

Caswell, H., R. J. Naiman, and R. Morin. 1984. Evaluating the consequences of reproduction in complex salmonid life cycles. Aquaculture 43:123-134.

Connor, E. F., S. H. Faeth, and D. Simberloff. 1983. Leafminers on oak: the role of immigration and in situ reproductive recruitment. Ecology 64:191-204.

Cormack, R. M. 1964. Estimates of survival from the sighting of marked animals. Biometrika 51:429-438.

de Kroon, H., A. Plaisier, J. van Groenendael, and H. Caswell. 1986. Elasticity: the relative contribution of demographic parameters to population growth rate. Ecology 67:1427-1431.

Fisher, R. A. 1930. The genetical theory of natural selection. Clarendon, Oxford, UK.

Franklin, A. B., K. P. Burnham, G. C. White, R. G. Anthony, E. D. Forsman, C. Schwarz, J. D. Nichols, and J. Hines 1999. Range-wide status and trends in northern spotted owl populations. Colorado Cooperative Fish and Wildlife Research Unit Report, Fort Collins, Colorado, USA.

Gaillard, J.-M., M. Festa-Bianchet, and N. G. Yoccoz. 1998. Population dynamics of large herbivores: variable recruitment with constant adult survival. Trends in Ecology and Evolution 13:58-63.

Gould, W. R., and J. D.Nichols. 1998. Estimation of temporal variability of survival in animal populations. Ecology 79 : 2531-2538.

Hestbeck, J., J. D. Nichols, and R. A. Malecki. 1991. Estimates of movement and site fidelity using mark-resight data of wintering Canada geese. Ecology 72:523-533.

Hines, J. E. 1994. MSSURVIV user's manual. Patuxent Environmental Science Center, Laurel, Maryland, USA.

Johnson, D. H., J. D. Nichols, and M. D. Schwarz. 1992. Population dynamics of breeding waterfowl. Pages 446-485 in B. D. J. Batt, A. D. Afton, M. G. Anderson, C. D. Ankney, D. H. Johnson, J. A. Kadlec, and G. L. Krapu, editors. Ecology and management of breeding waterfowl. University of Minnesota Press, Minneapolis, Minnesota, USA.

Jolly, G. M. 1965. Explicit estimates from capture-recapture data with both death and immigration-stochastic model. Biometrika 52:225-247.

Kendall, W. L., J. D. Nichols, and J. E. Hines. 1997. Estimating temporary emigration using capture-recapture data with Pollock's robust design. Ecology 78:563-578.

Kendall, W. L., K. H. Pollock, and C. Brownie. 1995. A likelihood-based approach to capture-recapture estimation of demographic parameters under the robust design. Biometrics 51:293-308.

Kuno, E. 1971. Sampling error as a misleading artifact in "key factor analysis." Researches on Population Ecology 13:28-45.

Lebreton, J.-D. 1996. Demographic models for subdivided populations: the renewal equation approach. Theoretical Population Biology 49:291-313.

Lebreton, J.-D., K. P. Burnham, J. Clobert, and D. R. Anderson. 1992. Modeling survival and testing biological hypotheses using marked animals: a unified approach with case studies. Ecological Monographs 62:67-118. 
Lee, S.-M., and A. Chao. 1994. Estimating population size via sample coverage for closed capture-recapture models. Biometrics 50:88-97.

Leslie, P. H. 1945. On the use of matrices in certain population mathematics. Biometrika 33:183-212.

Leslie, P. H. 1966. The intrinsic rate of increase and the overlap of successive generations in a population of guillemots (Uria aalge Pont.). Journal of Animal Ecology 35 : 291-301.

Lewis, E. G. 1942. On the generation and growth of a population. Sankhya 6:93-96.

Lewontin, R. C. 1965. Selection for colonizing species. Pages 77-94 in H. G. Baker and G. L. Stebbins, editors. The genetics of colonizing species. Academic Press, New York, New York, USA.

Link, W. A., and J. D.Nichols. 1994. On the importance of sampling variance to investigations of temporal variation in animal population size. Oikos 69:539-544.

Manly, B. F. J. 1977. The determination of key factors from life table data. Oecologia 31:111-117.

Manly, B. F. J. 1979. A note on key factor analysis. Researches on Population Ecology 21:30-39.

Menkins, G. E., Jr., and S. H. Anderson. 1988. Estimation of small-mammal population size. Ecology 69:1952-1959.

Mertz, D. B. 1971. The mathematical demography of the California condor population. American Naturalist 105: 437-453.

Morris, R. F. 1959. Single-factor analysis in population dynamics. Ecology 40:580-588.

Munro, R. E. and C. F. Kimball. 1982. Population ecology of the mallard VII. Distribution and derivation of the harvest U.S. Fish and Wildlife Service Resource Publication 147

Nichols, J. D., J. E. Hines, and K. H. Pollock. 1984b. Effects of permanent trap response in capture probability on JollySeber capture-recapture model estimates. Journal of Wildlife Management 48:289-294.

Nichols, J. D., J. E. Hines, K. H. Pollock, R. L. Hinz, and W. A. Link. 1994. Estimating breeding proportions and testing hypotheses about costs of reproduction with capture-recapture data. Ecology 75:2052-2065.

Nichols, J. D., R. W. Morris, C. Brownie, and K. H. Pollock. 1986. Sources of variation in extinction rates, turnover and diversity of marine invertebrate families during the Paleozoic. Paleobiology 12:421-432.

Nichols, J. D., and K. H. Pollock. 1990. Estimation of recruitment from immigration vs. in situ reproduction using Pollock's robust design. Ecology 71:21-26.

Nichols, J. D., K. H. Pollock, and J. E. Hines. 1984a. The use of a robust capture-recapture design in small mammal population studies: a field example with Microtus pennsylvanicus. Acta Theriologica 30:357-365.

Otis, D. L., K. P. Burnham, G. C. White, and D. R. Anderson. 1978. Statistical inference from capture data on closed animal populations. Wildlife Monographs No. 62.

Pollock, K. H. 1981. Capture-recapture models allowing for age-dependent survival and capture rates. Biometrics 37: 521-529.

Pollock, K. H. 1982. A capture-recapture sampling design robust to unequal catchability. Journal of Wildlife Management 46:752-757.

Pollock, K. H., J. E. Hines, and J. D. Nichols. 1985. Goodness-of-fit tests for open capture-recapture models. Biometrics 41:399-410.

Pollock, K. H., J. D. Nichols, C. Brownie, and J. E. Hines.
1990. Statistical inference for capture-recapture experiments. Wildlife Monographs No. 107.

Pollock, K. H., D. L. Solomon, and D. S. Robson. 1974. Tests for mortality and recruitment in a $K$-sample tag-recapture experiment. Biometrics 30:77-87.

Pradel, R. 1996. Utilization of capture-mark-recapture for the study of recruitment and population growth rate. Biometrics 52:703-709.

Pulliam, H. R. 1988. Sources, sinks, and population regulation. American Naturalist 132:652-661.

Rexstad, E., and K. P. Burnham. 1991. User's guide for interactive program CAPTURE. Abundance estimation of closed animal populations. Colorado State University, Fort Collins, Colorado, USA.

Reynolds, R. E., and J. R. Sauer. 1991. Changes in mallard breeding populations in relation to production and harvest rates. Journal of Wildlife Management 55:483-487.

Rogers, A. 1966. The multiregional matrix growth operator and the stable interregional age structure. Demography 3: 537-544.

Rose, R. K. 1973. A small mammal live trap. Transactions of the Kansas Academy of Science 76:14-17.

Roughgarden, J., S. Gaines, and H. Possingham. 1988. Recruitment dynamics in complex life cycles. Science 241: 1460-1466

Roughgarden, J., Y. Iwasa, and C. Baxter. 1985. Demographic theory for an open marine population with space-limited recruitment. Ecology 66:54-67.

Schoen, R. 1988. Modeling multigroup populations. Plenum, New York, New York, USA.

Schwarz, C. J., and A. N. Arnason. 1990. Use of tag-recovery information in migration and movement studies. American Fisheries Society Symposium 7:588-603.

Schwarz, C. J., J. F. Schweigert, and A. N. Arnason. 1993. Estimating migration rates using tag recovery data. Biometrics 49:177-193.

Schwarz, C. J., and W. T. Stobo. 1997. Estimating temporary migration using the robust design. Biometrics 53:178-194.

Seber, G. A. F. 1965. A note on the multiple-recapture census. Biometrika 52:249-259.

Seber, G. A. F. 1982. Estimation of animal abundance and related parameters, second edition. MacMillan, New York, New York, USA.

Skalski, J. R., and D. S. Robson. 1992. Techniques for wildlife investigations: design and analysis of capture data. Academic Press, San Diego, California, USA.

Tuljapurkar, S. 1990. Population dynamics in variable environments. Springer-Verlag, New York, New York, USA.

van Groenendael, J., H. de Kroon, and H. Caswell. 1988. Projection matrices in population biology. Trends in Ecology and Evolution 3:264-269.

Varley, G. C., and G. R. Gradwell. 1960. Key factors in population studies. Journal of Animal Ecology 29:399401

White, G. C. 1983. Numerical estimation of survival rates from band-recovery and biotelemetry data. Journal of Wildlife Management 47:716-728.

White, G. C., and K. P. Burnham. 1999. Program MARK: survival rate estimation from both live and dead encounters. Bird Study 46:(supplement)S120-139.

Yoccoz, N. G., H. Steen, R. A. Ims, and N. C. Stenseth. 1993. Estimating demographic parameters and the population size: an updated methodological survey. Pages 565-587 in N. C. Stenseth and R. A. Ims, editors. The biology of lemmings. The Linnean Society of London, London, UK.

\section{SUPPLEMENTARY MATERIAL}

The data set and analysis of the study of adult and subadult voles are available online in ESA's Ecological Archives: E081029 